\title{
Displacement of the Urinary Catheter Mimicking Bladder Rupture
}

\author{
Dong Hun Kim, M.D., Jeongseok Yun, M.D., Ph.D.
}

Department of Surgery, Trauma Center, Dankook University Hospital, Cheonan, Korea
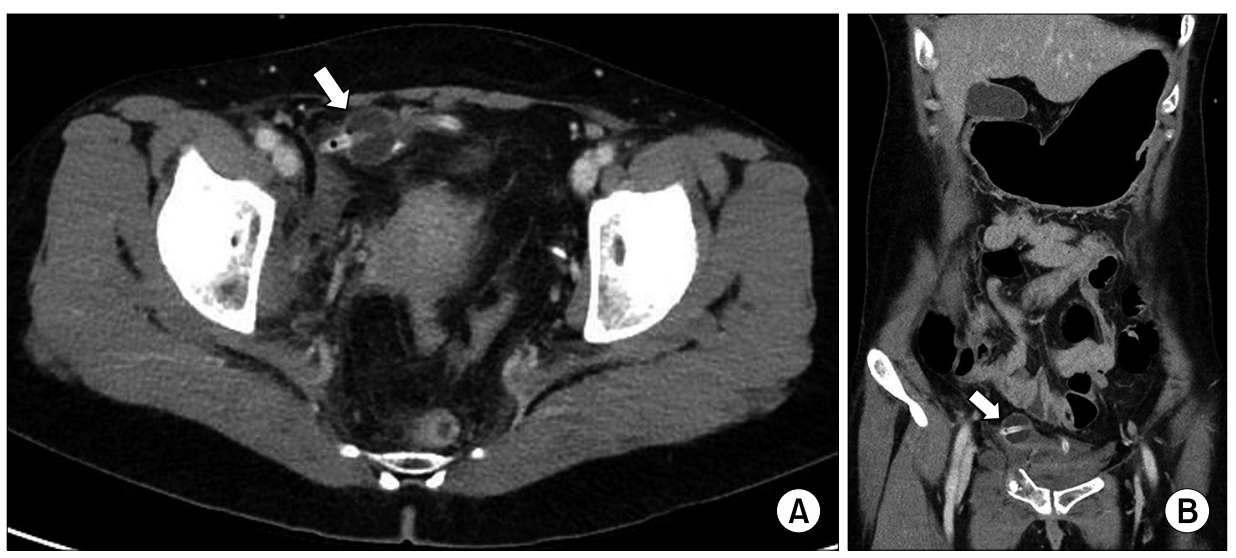

Fig. 1. Abdominal computed tomography shows a Foley catheter tip (white arrows) seen as migrating into an intraperitoneal space from bladder adjacent to the fracture of the right superior ramus with extraperitoneal hematoma. (A) Axial view and (B) Coronal view.

A 46-year-old woman had multiple pelvic fractures after blunt trauma. Physical examination revealed no peritoneal irritation sign except for suprapubic tenderness with gross hematuria. Computed tomography (CT) revealed that a Foley catheter seemed like migrating into an intraperitoneal space without intraperitoneal fluid collection (Fig. 1). And then, retrograde cystography followed for the definite diagnosis of bladder injury and demonstrated normal integrity of the bladder (Fig. 2). It seems like that the displaced Foley catheter within the collapsed bladder have confused as positioning out of bladder. Patients with disruption of the pubic symphysis, pubic rami, or an unstable pelvic fracture have a high incidence of concomitant bladder trauma [1]. CT in diagnosing traumatic bladder

Received April 19, 2018,

Revised July 23, 2018,

Accepted July 24, 2018

Correspondence to: Dong Hun Kim, M.D.

Department of Surgery, Trauma Center,

Dankook University Hospital, 201

Manghyang-ro, Dongnam-gu, Cheonan

31116, Korea

Tel: +82-41-550-7661, Fax: +82-41-550-0039

E-mail: saint7331@gmail.com

ORCID:

https://orcid.org/0000-0001-6613-6902 rupture is low accuracy compared with retrograde cystography [2]. A correct procedure, such as gently pulling a urinary catheter until inflation balloon is snug against the bladder neck, might prevent the unnecessary study like this case.

\section{Conflicts of Interest}

No potential conflict of interest relevant to this article was reported.

Copyright (C) 2019 by Korean Society of Acute Care Surgery

(c) This is an Open Access article distributed under the terms of the Creative Commons Attribution Non-Commercial License (http://creativecommons.org/licenses/by-nc/4.0) which permits unrestricted non-commercial use, distribution, and reproduction in any medium, provided the original work is properly cited. 


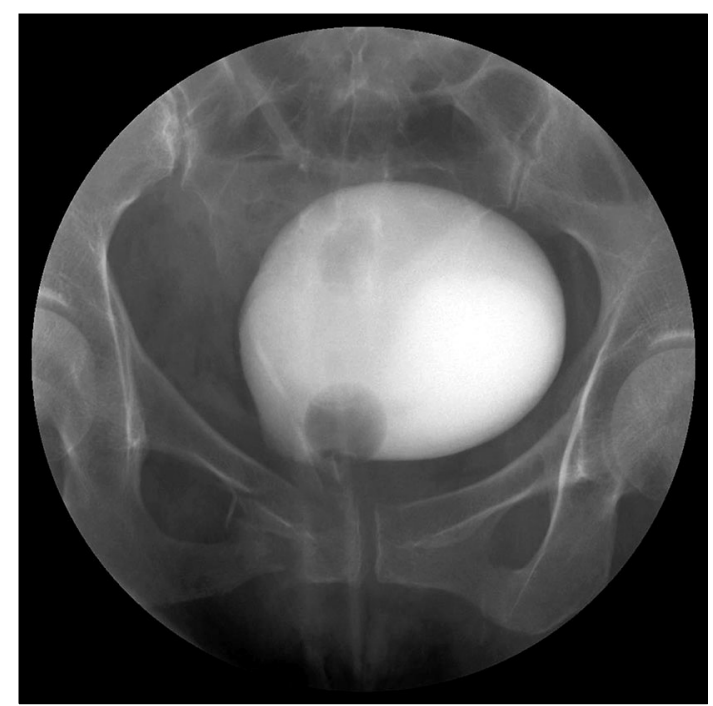

Fig. 2. A retrograde cystography shows normal distension of the urinary bladder filled with contrast and the balloon of the urinary catheter placed in bladder.

\section{References}

1. Figler BD, Hoffler CE, Reisman W, Carney KJ, Moore T, Feliciano D. Multi-disciplinary update on pelvic fracture associated bladder and urethral injuries. Injury 2012;43:1242-9.

2. Haas CA, Brown SL, Spirnak JP. Limitations of routine spiral computerized tomography in the evaluation of bladder trauma. J Urol 1999;162:51-2. 